\title{
Fuzzy Risk Analysis based on A New Approach of Ranking Fuzzy Numbers using Orthocenter of Centroids
}

\author{
N.Ravi Shankar \\ Dept. of Applied Mathematics \\ Mohd Lazim Abdullah \\ GIS, GITAM University \\ Dept. of Mathematics \\ Visakhapatnam, India
}

\author{
Y.L.P. Thorani \\ Dept. of Applied Mathematics \\ GIS, GITAM University \\ Visakhapatnam, India
}

\author{
P.Phani Bushan Rao \\ Dept. of Mathematics \\ GIT, GITAM University \\ Visakhapatnam, India
}

\begin{abstract}
In this paper, a new approach of ranking fuzzy numbers using orthocenter of centroids of fuzzy numbers to its distance from original point is proposed. The proposed method can rank all types of fuzzy numbers including crisp numbers with different membership functions. We apply the proposed ranking method to develop a new method to deal with fuzzy risk analysis problems. The proposed method is more flexible than the existing methods.
\end{abstract}

\section{Keywords}

Ranking fuzzy numbers; centroid; orthocenter; fuzzy ranking; fuzzy risk analysis.

\section{INTRODUCTION}

Ranking fuzzy numbers is an important tool in decision making. In fuzzy decision analysis, fuzzy quantities are used to describe the performance of alternatives in modeling a real-world problem. Most of the ranking procedures proposed so far in literature $[28,32]$ cannot discriminate fuzzy quantities and some are counterintuitive. As fuzzy numbers are represented by possibility distributions, they may overlap with each other and hence it is not possible to order them. It is true that fuzzy numbers are frequently partial order and cannot be compared like real numbers which can be linearly ordered. In order to rank fuzzy quantities, each fuzzy quantity is converted into a real number and compared by defining a ranking function from the set of fuzzy numbers to a set of real numbers which assigns a real number to each fuzzy number where a natural order exists. Usually by reducing the whole of any analysis to a single number, much of the information is lost and hence an attempt is to be made to minimize this loss.

Various ranking procedures have been developed since 1976 when the theory of fuzzy sets was first introduced by Zadeh [1]. Ranking fuzzy numbers was first proposed by
Jain [2] for decision making in fuzzy situations by representing the ill-defined quantity as a fuzzy set. Since then, various procedures to rank fuzzy quantities are proposed by various researchers. Bortolan and Degani [12] reviewed some of these ranking methods [2-11] for ranking fuzzy subsets. Chen [13] presented ranking fuzzy numbers with maximizing set and minimizing set. Dubois and Prade [16] presented the mean value of a fuzzy number. Lee and $\mathrm{Li}$ [18] presented a comparison of fuzzy numbers based on the probability measure of fuzzy events. Delgado, Verdegay and Vila [19] presented a procedure for ranking fuzzy numbers. Campos and Munoz [20] presented a subjective approach for ranking fuzzy numbers. Kim and Park [21] presented a method of ranking fuzzy numbers with index of optimism. Yuan [22] presented a criterion for evaluating fuzzy ranking methods. Heilpern [23] presented the expected value of a fuzzy number. Saade and Schwarzlander [24] presented ordering fuzzy sets over the real line. Liou and Wang [25] presented ranking fuzzy numbers with integral value. Choobineh and $\mathrm{Li}$ [26] presented an index for ordering fuzzy numbers. Since then several methods have been proposed by various researchers which include ranking fuzzy numbers using area compensation, distance method, decomposition principle and signed distance [27, 28, 29]. Wang and Kerre [30, 31] classified all the above ranking procedures into three classes. The first class consists of ranking procedures based on fuzzy mean and spread [6, 7, 8, 9, 20, 25, 26, 27] and second class consists ranking procedures based on fuzzy scoring $[2,4,10,13,17,21]$ whereas the third class consists of methods based on preference relations $[3,5,11$, $14,15,19,22,24]$ and concluded that the ordering procedures associated with first class are relatively reasonable for the ordering of fuzzy numbers specially the ranking procedure presented by Adamo [7] which satisfies all the reasonable properties for the ordering of fuzzy quantities. The methods presented in the second class are not doing well and the methods $[14,15,22,24]$ which belong to class three are reasonable. Later on, ranking 
fuzzy numbers by preference ratio [32], left and right dominance [33], area between the centroid point and original point [34], ], sign distance [36], distance minimization [37] came into existence. Later in 2007, Garcia and Lamata [38] modified the index of Liou and Wang [25] for ranking fuzzy numbers, by stating that the index of optimism is not alone sufficient to discriminate fuzzy numbers and proposed an index of modality to rank fuzzy numbers. Most of the methods [28,34] presented above cannot discriminate fuzzy numbers and some methods do not agree with human intuition whereas, some methods cannot rank crisp numbers which are a special case of fuzzy numbers.

One of the possible methods to overcome these problems is to introduce ranking based on Orthocentre of centroids to rank fuzzy quantities. In a trapezoidal fuzzy number, first the trapezoid is split into three parts where the first, second and third parts are a triangle, a rectangle and a triangle respectively. Then the centroids of these three parts are calculated followed by the calculation of the orthocentre of these centroids. Orthocentre is the point where the three altitudes of a triangle intersect. The altitude of a triangle is a line which passes through a vertex of a triangle and is perpendicular to the opposite side. Finally, a ranking function is defined which is the Euclidean distance between the centroid point and the original point to rank fuzzy numbers. Most of the ranking procedures proposed in literature use Centroid of trapezoid as reference point, as the Centroid is a balancing point of the trapezoid. But the Orthocenter of centroids can be considered a much more balancing point than the centroid. Further, this method uses an index of optimism to reflect the decision maker's optimistic attitude and also uses an index of modality that represents the neutrality of the decision maker.

The work is organized as follows: Section 2 briefly introduces the basic concepts and definitions of fuzzy numbers. Section 3 presents the proposed new ranking method. In Section 4, the proposed method has been explained with examples which describe the advantages and the efficiency of the method which ranks generalized fuzzy numbers, images of fuzzy numbers and even crisp numbers. In Section 5, the method demonstrates its robustness by comparing with other methods like Liou and Wang[25], Yager[8] and others where the methods cannot discriminate fuzzy quantities and do not agree with human intuition. In Section 6, the proposed ranking method is applied to propose a fuzzy risk algorithm to deal with fuzzy risk analysis problems. Finally, the conclusions of the work are presented in Section 7.

\section{FUZZY CONCEPTS AND RANKING OF FUZZY NUMBERS}

\subsection{Fuzzy concepts}

In this section, some fuzzy basic definitions are presented [42].

Definition 1. Let $U$ be a Universe set. A fuzzy set $\tilde{\mathrm{A}}$ of $U$ is defined by a membership function $\mathrm{f}_{\tilde{\mathrm{A}}}: \mathrm{U} \rightarrow[0,1]$, where $\mathrm{f}_{\tilde{\mathrm{A}}}(\mathrm{x})$ is the degree of $x$ A

in $\mathrm{A}, \forall \mathrm{x} \in \mathrm{U}$.

Definition 2. A fuzzy set $\tilde{A}$ of Universe set $U$ is normal if and only if $\sup _{\mathrm{x} \in \mathrm{U}} \mathrm{f}_{\tilde{\mathrm{A}}}(\mathrm{x})=1$

Definition 3. A fuzzy set $\tilde{A}$ of Universe set $U$ is convex if and only

$f_{\tilde{A}}(\lambda x+(1-\lambda) y) \geq \min \left(f_{\tilde{A}}(x), f_{\tilde{A}}(y)\right)$,

$\forall x, y \in U$ and $\lambda \in[0,1]$.

Definition 4. A fuzzy set $\tilde{\mathrm{A}}$ of Universe set $U$ is a fuzzy number iff $\tilde{A}$ is normal and convex on $U$.

Definition 5. A real fuzzy number $\tilde{A}$ is described as any fuzzy subset of the real line $\tilde{A}$ with membership function $\mathrm{f}_{\tilde{\mathrm{A}}}(\mathrm{x})$ possessing the following properties:

(1) $\mathrm{f}_{\tilde{\mathrm{A}}}(\mathrm{x})$ is a continuous mapping from $\mathfrak{R}$ to the closed interval $[0, \mathrm{w}] .0<\mathrm{w} \leq 1$

(2) $\mathrm{f}_{\tilde{\mathrm{A}}}(\mathrm{x})=0$, for all $x \in(-\infty, a]$

(3) $\mathrm{f}_{\tilde{\mathrm{A}}}(\mathrm{x})$ is strictly increasing on $[a, b]$

(4) $\mathrm{f}_{\tilde{\mathrm{A}}}(\mathrm{x})=1$, for all $x \in[b, c]$

(5) $\mathrm{f}_{\tilde{\mathrm{A}}}(\mathrm{x})$ is strictly decreasing on $[c, d]$

(6) $\mathrm{f}_{\tilde{\mathrm{A}}}(\mathrm{x})=0$, for all $x \in(d, \infty]$, where $a, b, c, d$ are real numbers. 
Definition 6. The membership function of the real fuzzy number $\tilde{A}$ is given by

$$
f_{\tilde{A}}(x)=\left\{\begin{array}{c}
f_{\tilde{A}}^{L}, \quad a \leq x \leq b, \\
w, \quad b \leq x \leq c, \\
f_{\tilde{A}}^{R}, \quad c \leq x \leq d, \\
0, \quad \text { otherwise, }
\end{array}\right.
$$

where $0<w \leq 1$ is a constant, $a, b, c, d \quad$ are real numbers $\quad$ and $\quad f_{\tilde{A}}^{L}:[a, b] \rightarrow[0, w]$, $f_{\tilde{A}}^{R}:[c, d] \rightarrow[0, w]$ are two strictly monotonic and continuous functions from $\mathfrak{R}$ to the closed interval $[0, w]$. It is customary to write a fuzzy number as

$$
\tilde{A}=(a, b, c, d ; w) . \quad \text { If } w=1,
$$

then $A=(a, b, c, d ; 1)$ is a normalized fuzzy number,

otherwise $\tilde{A}$ is said to be a generalized or non-normal fuzzy number. If the membership function $f_{\tilde{A}}(x)$ is

piecewise linear, then $\tilde{A}$ is said to be a trapezoidal fuzzy number. The membership function of a trapezoidal fuzzy number is given by:

$$
f_{\tilde{A}}(x)= \begin{cases}\frac{w(x-a)}{b-a}, & a \leq x \leq b, \\ w, & b \leq x \leq c, \\ \frac{w(x-d)}{c-d}, & c \leq x \leq d, \\ 0, & \text { otherwise, }\end{cases}
$$

If $w=1$, then $\tilde{A}=(a, b, c, d ; 1)$ is a normalized trapezoidal fuzzy number and $\tilde{A}$ is a generalized or non normal trapezoidal fuzzy number if $0<w<1$.

The image of $\tilde{A}=(a, b, c, d ; w)$ is given by $-\tilde{A}=(-d,-c,-b,-a ; w)$. As a particular case if $b=c$, the trapezoidal fuzzy number reduces to a triangular fuzzy number given by $\tilde{A}=(a, b, d ; w)$. The value of ' $b$ ' corresponds with the mode or core and $[a, d]$ with the support. If $w=1$, then $\tilde{A}=(a, b, d)$ is a normalized triangular fuzzy number. $\tilde{A}$ is a generalized or non normal triangular fuzzy number if $0<w<1$. As $f_{\tilde{A}}^{L}:[a, b] \rightarrow[0, w]$ and
$f_{\tilde{A}}^{R}:[c, d] \rightarrow[0, w] \quad$ are strictly monotonic and continuous functions, their inverse functions $g_{\tilde{A}}^{L}:[0, w] \rightarrow[a, b]$ and $\quad g_{\tilde{A}}^{R}:[0, w] \rightarrow[c, d]$ are also continuous and strictly monotonic. Hence $g_{\tilde{A}}^{L}$ and $g_{\tilde{A}}^{R}$ are integrable on $[0, w]$.In this paper, we use fuzzy arithmetical operators shown in (i)-(iv) to deal with the fuzzy arithmetical operations between generalized fuzzy numbers. Assume that there are two generalized trapezoidal fuzzy numbers $\tilde{A}_{1}$ and $\widetilde{A}_{2}$ where $\tilde{A}_{1}=\left(a_{1}, b_{1}, c_{1}, d_{1} ; w_{1}\right)$ and $\tilde{A}_{2}=\left(a_{2}, b_{2}, c_{2}, d_{2} ; w_{2}\right)$. The arithmetic operations between the generalized trapezoidal fuzzy numbers $\tilde{A}_{1}$ and $\tilde{A}_{2}$ are reviewed from [41],[42] and [43] as follows :

(i) Fuzzy Numbers Addition $\oplus$

$$
\tilde{\mathrm{A}}_{1} \oplus \tilde{\mathrm{A}}_{2}=
$$

$\left(a_{1}, b_{1}, c_{1}, d_{1} ; w_{1}\right) \oplus\left(a_{2}, b_{2}, c_{2}, d_{2} ; w_{2}\right)$

$=\left(a_{1}+a_{2}, b_{1}+b_{2}, c_{1}+c_{2}, d_{1}+d_{2} ; \min \left(w_{1}, w_{2}\right)\right)$ where $a_{1}, b_{1}, c_{1}, d_{1}, a_{2}, b_{2}, c_{2}$ and $d_{2}$ are any real numbers.

(ii)Fuzzy Numbers subtraction $\Theta$

$$
\begin{aligned}
& \tilde{\mathrm{A}}_{1} \Theta \tilde{\mathrm{A}}_{2}= \\
& \quad\left(a_{1}, b_{1}, c_{1}, d_{1} ; w_{1}\right) \Theta\left(a_{2}, b_{2}, c_{2}, d_{2} ; w_{2}\right)
\end{aligned}
$$

$=\left(a_{1}-d_{2}, b_{1}-c_{2}, c_{1}-b_{2}, d_{1}-a_{2} ; \min \left(w_{1}, w_{2}\right)\right)$ where $a_{1}, b_{1}, c_{1}, d_{1}, a_{2}, b_{2}, c_{2}$ and $d_{2}$ are any real numbers .

(iii) Fuzzy Numbers Multiplication $\otimes$

$$
\tilde{\mathrm{A}}_{1} \otimes \tilde{\mathrm{A}}_{2}=\left(a, b, c, d ; \min \left(w_{1}, w_{2}\right)\right)
$$

where,

$$
\begin{aligned}
& a=\operatorname{Min}\left(a_{1} \times a_{2}, a_{1} \times d_{2}, d_{1} \times a_{2}, d_{1} \times d_{2}\right), \\
& b=\operatorname{Min}\left(b_{1} \times b_{2}, b_{1} \times c_{2}, c_{1} \times b_{2}, c_{1} \times c_{2}\right), \\
& c=\operatorname{Max}\left(b_{1} \times b_{2}, b_{1} \times c_{2}, c_{1} \times b_{2}, c_{1} \times c_{2}\right), \\
& \text { and } d=\operatorname{Min}\left(a_{1} \times a_{2}, a_{1} \times d_{2}, d_{1} \times a_{2}, d_{1} \times d_{2}\right) .
\end{aligned}
$$

It is obvious that if $a_{1}, b_{1}, c_{1}, d_{1}, a_{2}, b_{2}, c_{2}, d_{2}$ are all positive-real numbers, then

$$
\begin{aligned}
& \tilde{\mathrm{A}}_{1} \otimes \tilde{\mathrm{A}}_{2}= \\
& \left(a_{1} \times a_{2}, b_{1} \times b_{2}, c_{1} \times c_{2}, d_{1} \times d_{2} ; \min \left(w_{1}, w_{2}\right)\right) .
\end{aligned}
$$

(iv) Fuzzy Numbers Division $\varnothing$ : 
The inverse of the fuzzy number $\widetilde{\mathrm{A}}_{2}$ is

$$
\frac{1}{\widetilde{A}_{2}}=\left(\frac{1}{d_{2}}, \frac{1}{c_{2}}, \frac{1}{b_{2}}, \frac{1}{a_{2}} ; w_{2}\right)
$$

where $a_{2}, b_{2}, c_{2}$ and $d_{2}$ are all real numbers except zero .Let $a_{1}, b_{1}, c_{1}, d_{1}, a_{2}, b_{2}, c_{2}$ and $d_{2}$ be non-zero positive real numbers . Then ,the division of $\widetilde{\mathrm{A}}_{1}$ and $\widetilde{\mathrm{A}}_{2}$ is as follows:

$$
\tilde{\mathrm{A}}_{1} \varnothing \tilde{\mathrm{A}}_{2}=\left(\frac{a_{1}}{d_{2}}, \frac{b_{1}}{c_{2}}, \frac{c_{1}}{b_{2}}, \frac{d_{1}}{a_{2}} ; \min \left(w_{1}, w_{2}\right)\right)
$$

\subsection{Review of some ranking fuzzy numbers}

\subsubsection{Cheng's Ranking method}

Cheng [28] ranked fuzzy numbers with the distance method using the Euclidean distance between the Centroid point and original point. For a generalized Trapezoidal fuzzy

number $\tilde{A}=(a, b, c, d ; w)$. the centroid is given by:

$\left(\overline{\mathrm{x}}_{0}, \overline{\mathrm{y}}_{0}\right)=$

$\left(\frac{w\left(d^{2}-2 c^{2}+2 b^{2}-a^{2}+d c-a b\right)+3\left(c^{2}-b^{2}\right)}{3 w(d-c+b-a)+6(c-b)}\right.$,

$\left.\frac{w}{3}\left(1+\frac{(b+c)-(a+d)(1-w)}{(b+c-a-d)+2(a+d) w}\right)\right)$

and the ranking function $\widetilde{\mathrm{A}}_{\mathrm{i}}>\widetilde{\mathrm{A}}_{\mathrm{j}}$ associated with $\tilde{A}$

as $R(\tilde{A})=\sqrt{\bar{x}_{0}^{2}+\bar{y}_{0}^{2}}$

Let $\widetilde{A}_{i}$ and $\tilde{A}_{j}$ be two fuzzy numbers,

(i) If $R\left(\tilde{A}_{i}\right)>R\left(\tilde{A}_{j}\right)$ then $\tilde{A}_{i}>\tilde{A}_{j}$.

(ii)If $R\left(\tilde{A}_{i}\right)<R\left(\tilde{A}_{j}\right)$ then $\tilde{A}_{i}<\tilde{A}_{j}$

(iii) If $R\left(\tilde{A}_{i}\right)=R\left(\tilde{A}_{j}\right)$ then $\tilde{A}_{i}=\tilde{A}_{j}$

He further improved Lee and Li's method by proposing the index of coefficient of variation $(\mathrm{CV})$ as $C V=\frac{\sigma}{|\mu|}$ where $\sigma$ is standard error and $\mu$ is mean, $\mu \neq 0$ and $\sigma>0$, the fuzzy number with smaller $C V$ is ranked higher.

\subsubsection{Wang et al. Ranking method}

Wang et al. [35] found that the centroid formulae proposed by Cheng [28] are incorrect and have led to some misapplications such as by Chu and Tsao [34]. They presented the correct centroid formulae, for a generalized fuzzy number $\tilde{A}=(a, b, c, d ; w)$ as:

$$
\begin{aligned}
& O\left(\bar{x}_{0}, \bar{y}_{0}\right)=\left(a+b+c+d-\frac{d c-a b}{(d+c)-(a+b)}\right. \\
& \left.\frac{w}{3}\left(1+\frac{c-b}{(d+c)-(a+b)}\right)\right) \\
& \text { and the ranking function associated with } \tilde{A}^{-} \\
& \text {as } R(\tilde{A})=\sqrt{\bar{x}_{0}^{2}+\bar{y}_{0}^{2}} \text {. } \\
& \text { Let } \tilde{A}_{i} \text { and } \tilde{A}_{j} \text { two fuzzy numbers, } \\
& \text { (i) If } R\left(\tilde{A}_{i}\right)>R\left(\tilde{A}_{j}\right) \text { then } \tilde{A}_{i}>\tilde{A}_{j} \\
& \text { (ii) If } R\left(\tilde{A}_{i}\right)<R\left(\tilde{A}_{j}\right) \text { then } \tilde{A}_{i}<\tilde{A}_{j} \\
& \text { (iii) If } R\left(\tilde{A}_{i}\right)=R\left(\tilde{A}_{j}\right) \text { then } \tilde{A}_{i}=\tilde{A}_{j}
\end{aligned}
$$

\subsubsection{Liou and Wang's Ranking method}

Liou and Wang [25] ranked fuzzy numbers with total integral value. For a fuzzy number defined

by definition 6 the total integral value is defined as $I_{T}^{\alpha}(\tilde{A})=\alpha I_{R}(\tilde{A})+(1-\alpha) I_{L}(\tilde{A}) \quad$ where $I_{R}(\tilde{A})=\int_{0}^{1} g_{\tilde{A}}^{R}(y) d y$ and $I_{L}(\tilde{A})=\int_{0}^{1} g_{\tilde{A}}^{L}(y) d y \quad$ are the right and left integral values of $\widetilde{A}$, respectively and $\alpha \in[0,1]$ is the index of optimism which represents the degree of optimism of a decision maker. If $\alpha=0$ the total integral value represents a pessimistic decision maker's view point which is equal to left integral value. If $\alpha=1$, the total integral value represents an optimistic decision maker's view point and is equal to the right integral value and when $\alpha=0.5$, the total integral value represents an moderate decision maker's view point and is equal to the mean of right and left integral values. For a decision maker, the larger the value of $\alpha$ is, the higher is the degree of optimism.

\subsubsection{Garcia and Lamata's Ranking method}

Garcia and Lamata [38] modified the index of Liou and Wang [25] for ranking fuzzy numbers. This method use an index of optimism to reflect the decision maker's optimistic attitude, which is not enough to discriminate fuzzy numbers, but rather it also uses an index of modality that represents the neutrality of the decision maker. For a fuzzy number defined by definition 6, Garcia and Lamata [38] proposed an index associated with the ranking as the convex combination: $I_{\beta, \alpha}(\tilde{A})=\beta S_{M}(\tilde{A})+(1-\beta) I_{T}^{\alpha}(\tilde{A})$, where $S_{M}(\tilde{A})$ is the area of the core of the fuzzy number which is equal to ' $b$ ' for a triangular fuzzy number defined by $\tilde{A}=(a, b, d ; w)$. and the average value of the plateau in case of a trapezoidal fuzzy number given 
by $\tilde{A}=(a, b, c, d ; w), \beta \in[0,1]$ is the index of modality that represents the importance of central value against the extreme values, $\alpha \in[0,1]$ is the degree of optimism of the decision maker and $I_{T}^{\alpha}(\tilde{A})$ has its own meaning as defined in section 2.2.3.

\section{PROPOSED RANKING METHOD}

The Centroid of a trapezoid is considered as the balancing point of the trapezoid (Fig.1). Divide the trapezoid into three plane figures. These three plane figures are a triangle (APB), a rectangle (BPQC), and a triangle (CQD), respectively. Let the Centroids of the three plane figures be $G_{1}, G_{2} \& G_{3}$ respectively. The orthocenter of these Centroids $G_{1}, G_{2} \& G_{3}$ is taken as the point of reference to define the ranking of generalized trapezoidal fuzzy numbers. The reason for selecting this point as a point of reference is that each Centroid point are balancing points of each individual plane figure, and the orthocentre of these Centroid points is a much more balancing point for a generalized trapezoidal fuzzy number. Orthocentre is the point where the three altitudes of a triangle intersect. The altitude of a triangle is a line which passes through a vertex of a triangle and is perpendicular to the opposite side. Therefore, this point would be a better reference point than the Centroid point of the trapezoid.

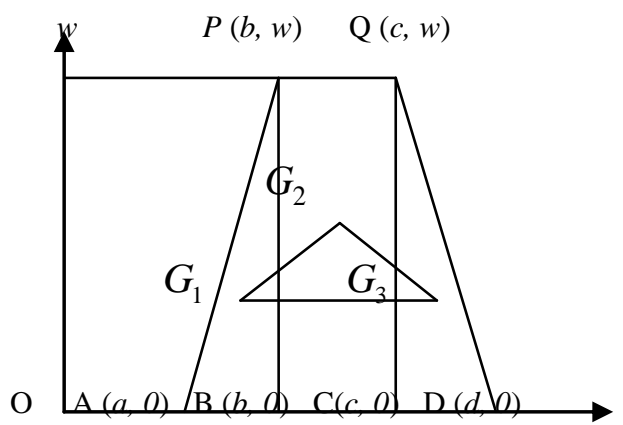

Fig. 1. Orthocenter of Centroids

Consider a generalized trapezoidal fuzzy number $\tilde{A}=(a, b, c, d ; w)$ (Fig.1). The Centroids of the three plane figures are $G_{1}=\left(\frac{a+2 b}{3}, \frac{w}{3}\right)$, $G_{2}=\left(\frac{b+c}{2}, \frac{w}{2}\right) \quad$ and $\quad G_{3}=\left(\frac{2 c+d}{3}, \frac{w}{3}\right)$ respectively. Equation of the line $\overleftrightarrow{G_{1} G_{3}}$ is $y=\frac{w}{3}$ and $G_{2}$ does not lie on the line $\overleftrightarrow{G_{1} G_{3}}$. Therefore, $\mathrm{G}_{1} \mathrm{G}_{2}$ and $\mathrm{G}_{3}$ are non-collinear and they form a triangle. We define the Orthocentre $O_{\tilde{A}}\left(\bar{x}_{0}, \bar{y}_{0}\right)$ of the triangle with vertices $G_{1}, G_{2}$ and $\mathrm{G}_{3}$ of the generalized trapezoidal fuzzy number $\tilde{A}=(a, b, c, d ; w)$ as

$$
\begin{aligned}
& O_{\tilde{A}}\left(\bar{x}_{0,} \bar{y}_{0}\right)=\left(\frac{1}{2}(c+b),\right. \\
& \left.\frac{(3 c-2 a-b)(c-3 b+2 d)+2 w^{2}}{6 w}\right)
\end{aligned}
$$

As a special case, for triangular fuzzy number $\tilde{A}=(a, b, d ; w)$. i.e., $c=b$ the Orthocentre of centroids is given by $O_{\tilde{A}}\left(\bar{x}_{0}, \bar{y}_{0}\right)=\left(b, \frac{(b-a)(d-b)+w^{2}}{3 w}\right)$

For a generalized trapezoidal fuzzy number $\tilde{A}=(a, b, c, d ; w)$., with orthocentre of centroids $O_{\tilde{A}}\left(\bar{x}_{0}, \bar{y}_{0}\right)$ defined by Eq. (3), we define the index associated with the ranking as $I_{\alpha}(\tilde{A})=\alpha \bar{y}_{0}+(1-\alpha) \bar{x}_{0} \quad$ where $\quad \alpha \in[0,1]$ is the index of optimism which represents the degree of optimism of a decision maker. If $\alpha=0$,we have a pessimistic decision maker's view point which is equal to the distance of the orthocentre from $y$-axis. If $\alpha=1$, we have an optimistic decision maker's view point and is equal to the distance of the orthocentre from $x$-axis, and when $\alpha=0.5$ we have the moderate decision maker's view point and is equal to the mean of the distances of orthocentre from $y$ and $x$ axes. The larger the value of $\alpha$ is, the higher the degree of optimism of the decision maker. The index of optimism is not alone sufficient to discriminate fuzzy numbers as this uses only the extreme values of the orthocentre of centroids. Hence, we upgrade this by using an index of modality which represents the importance of central value along with index of optimism. For a generalized trapezoidal fuzzy number $\tilde{A}=(a, b, c, d ; w)$, with orthocentre of centroids $O_{\tilde{A}}\left(\bar{x}_{0}, \bar{y}_{0}\right)$ defined by Eq.(3), we define the index associated with the ranking as $I_{\alpha, \beta}(\tilde{A})=\beta S_{M}(\tilde{A})+(1-\beta) I_{\alpha}(\tilde{A})$ where $\beta \in[0,1]$ is the index of modality which represents the importance of central value against the extreme values $\bar{x}_{0}$ and $\bar{y}_{0}, S_{M}(\tilde{A})$ is the mode associated with the fuzzy number which is equal to ' $b$ ' for a triangular fuzzy number $\tilde{A}=(a, b, d ; w)$. and the average value of the plateau for a trapezoidal fuzzy number and $I_{\alpha}(\tilde{A})$ is the one which is defined in definition 2 . Here $\beta$ represents the weight of the central value and $1-\beta$ is the weight associated with the extreme values $\bar{x}_{0}$ and $\bar{y}_{0}$. 
For any decision maker whether pessimistic ( $\alpha=0)$, optimistic ( $\alpha=1$ ) or neutral( $\alpha=0.5$ ), the ranking function of the trapezoidal fuzzy number $\tilde{A}=(a, b, c, d ; w)$. which maps the set of all fuzzy numbers to a set of real numbers is defined as $R(\tilde{A})=\sqrt{\bar{x}_{0}^{2}+\bar{y}_{0}^{2}}$ which is, the Euclidean distance from the orthocentre of the centroids as defined from the original point.

We define ranking between fuzzy numbers as: Let $\tilde{A}_{i}$ and $\tilde{A}_{j}$ be two fuzzy numbers, then case (i ) If $R\left(\tilde{A}_{i}\right)>R\left(\tilde{A}_{j}\right)$ then $\tilde{A}_{i}>\tilde{A}_{j}$ case ( ii ) If $R\left(\tilde{A}_{i}\right)<R\left(\tilde{A}_{j}\right)$ then $\tilde{A}_{i}<\tilde{A}_{j}$ case ( iii ) If $R\left(\tilde{A}_{i}\right)=R\left(\tilde{A}_{j}\right)$ then in this case the discrimination of fuzzy numbers is not possible.

In this case we use $I_{\alpha, \beta}(\tilde{A})=\beta S_{M}(\tilde{A})+(1-\beta) I_{\alpha}(\tilde{A}) \quad$ where $\beta \in[0,1]$ and the ranking has been done as follows :

(a) if $I_{\alpha, \beta}\left(\tilde{A}_{i}\right)>I_{\alpha, \beta}\left(\tilde{A}_{j}\right)$ then $\tilde{\mathrm{A}}_{\mathrm{i}}>\tilde{\mathrm{A}}_{\mathrm{j}}$ and

(b) if $I_{\alpha, \beta}\left(\tilde{A}_{i}\right)<I_{\alpha, \beta}\left(\tilde{A}_{j}\right)$ then $\tilde{A}_{i}>\tilde{A}_{j}$.

\section{NUMERICAL EXAMPLES}

\section{Example 4.1}

Let $\quad \widetilde{A}=(0,1,2 ; 1), \widetilde{B}=\left(\frac{1}{5}, 1, \frac{7}{4} ; 1\right) \quad$ be $\quad$ two normalized positive triangular fuzzy numbers.

Then $O_{\widetilde{A}}\left(\bar{x}_{0}, \bar{y}_{0}\right)=(1,0.6666)$

$O_{\widetilde{B}}\left(\bar{x}_{0}, \bar{y}_{0}\right)=(1,0.5333)$

Therefore, $\quad R(\tilde{A})=1.2018$ and $R(\tilde{B})=1.1333$

$\Rightarrow \tilde{A}>\widetilde{B}$

It is observed that the above ranking order is unaltered even by using the index of modality proposed in section 3 irrespective of the decision maker.

\section{Example 4.2}

Let $\widetilde{A}=(0.1,0.3,0.3,0.5 ; 1)$,

$\widetilde{B}=(-0.5,-0.3,-0.3,-0.1 ; 1)$ be two normalized trapezoidal fuzzy numbers with opposite sign.

Then

$$
\begin{aligned}
& O_{\widetilde{A}}\left(\bar{x}_{0}, \bar{y}_{0}\right)=(0.3,0.36) \text { and } \\
& O_{\widetilde{B}}\left(\bar{x}_{0}, \bar{y}_{0}\right)=(-0.3,0.36) .
\end{aligned}
$$

Therefore $\quad R(\tilde{A})=0.4686, R(\tilde{B})=0.4686 \quad$ and discrimination of fuzzy numbers is not possible. Now by using case (iii) as defined in section 3, we have

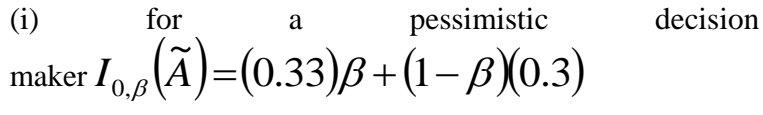

$I_{0, \beta}(\tilde{B})=(0.33) \beta+(1-\beta)(-0.3)$

as

$(0.33) \beta+(1-\beta)(0.3)>(0.33) \beta+(1-\beta)(-0.3)$

$\Rightarrow \tilde{A}>\tilde{B}$.

(ii) for a optimistic decision maker

$I_{1, \beta}(\tilde{A})=(0.33) \beta+(1-\beta)(0.36)$

$I_{1, \beta}(\tilde{B})=(0.03) \beta+(1-\beta)(0.36)$

as

$(0.33) \beta+(1-\beta)(0.36)>(0.03) \beta+(1-\beta)(0.36)$

$\Rightarrow \tilde{A}>\tilde{B}$

(iii) for a neutral decision maker

$I_{0.5, \beta}(\tilde{A})=(0.33) \beta+(1-\beta)(0.33)$

$I_{0.5, \beta}(\tilde{B})=(0.03) \beta+(1-\beta)(0.03)$

as

$(0.33) \beta+(1-\beta)(0.33)>(0.03) \beta+(1-\beta)(0.03)$

$\Rightarrow \tilde{A}>\tilde{B}$

Thus we see that the ranking order is same in all the three cases.

\section{Example 4.3}

Let $\widetilde{A}=(0.1,0.3,0.3,0.5 ; 1)$, and

$\widetilde{B}=(-0.5,-0.3,-0.3,-0.1 ; 1)$ be two trapezoidal fuzzy numbers in example 4.2 .

Then $-\tilde{A}=(-0.5,-0.3,-0.3,-0.1 ; 1)$ and

$-\widetilde{B}=(0.1,0.3,0.3,0.5 ; 1)$,

$O_{-\tilde{A}}\left(\bar{x}_{0}, \bar{y}_{0}\right)=(-0.3,0.36)$ and

$O_{-\tilde{B}}\left(\bar{x}_{0}, \bar{y}_{0}\right)=(0.3,0.36)$.

Therefore $R(-\tilde{A})=0.4686, R(-\tilde{B})=0.4686$ and discrimination of fuzzy numbers is not possible. We have (i)for an pessimistic decision maker

$$
\begin{aligned}
& I_{0, \beta}(-\tilde{A})=(0.03) \beta+(1-\beta)(-0.3) \\
& I_{0, \beta}(-\widetilde{B})=(0.33) \beta+(1-\beta)(0.3) \\
& \text { as }(0.33) \beta+(1-\beta)(0.3)>(0.03) \beta+(1-\beta)(-0.3) \\
& \Rightarrow-\tilde{A}<-\widetilde{B}
\end{aligned}
$$

(ii)for an optimistic decision maker 


$$
\begin{aligned}
& I_{1, \beta}(-\tilde{A})=(0.03) \beta+(1-\beta)(0.36) \\
& I_{1, \beta}(-\tilde{B})=(0.33) \beta+(1-\beta)(0.36) \\
& \text { as }(0.33) \beta+(1-\beta)(0.36)>(0.03) \beta+(1-\beta)(0.36) \\
& \Rightarrow-\tilde{A}<-\tilde{B} \\
& \text { (iii) } \quad \text { for a neutral decision maker } \\
& I_{0.5, \beta}(-\tilde{A})=(0.03) \beta+(1-\beta)(0.03) \\
& I_{0.5, \beta}(-\tilde{B})=(0.33) \beta+(1-\beta)(0.33) \\
& \text { as }(0.33) \beta+(1-\beta)(0.33)>(0.03) \beta+(1-\beta)(0.03) \\
& \Rightarrow-\tilde{A}<-\tilde{B}
\end{aligned}
$$

Thus we see that the ranking order is same in all the three cases.

From examples 4.2 and 4.3 we see that $\widetilde{A}>\widetilde{B} \Rightarrow-\tilde{A}<-\widetilde{B}$.

\section{Example 4.4}

Let $\quad \tilde{A}=(0.1,0.2,0.4,0.5 ; 1)$ be a normalized trapezoidal fuzzy number and $\widetilde{B}=(1,1,1,1 ; 1) \quad$ be $\quad$ a $\quad$ crisp number. Then $O_{\widetilde{A}}\left(\bar{x}_{0}, \bar{y}_{0}\right)=(0.3,0.44)$ and

$O_{\widetilde{B}}\left(\bar{x}_{0}, \bar{y}_{0}\right)=(1,0.3333)$.

Therefore,

$$
R(\tilde{A})=0.5325 \text { and } R(\tilde{B})=1.0440 \Rightarrow \tilde{A}<\tilde{B}
$$

It is observed that the above ranking order is unaltered even by using the index of modality proposed irrespective of the decision maker.

Example 4.5

Let $\widetilde{A}=(0.1,0.3,0.5 ; 0.8), \widetilde{B}=(0.1,0.3,0.5 ; 1)$ be two generalized trapezoidal fuzzy numbers.

$$
\begin{aligned}
& \text { Then } O_{\tilde{A}}\left(\bar{x}_{0}, \bar{y}_{0}\right)=(0.3,0.2833) \text { and } \\
& O_{\widetilde{B}}\left(\bar{x}_{0}, \bar{y}_{0}\right)=(0.3,0.3466) \\
& \text { Therefore, } R(\tilde{A})=0.4126 \text { and } \quad R(\tilde{B})=0.4583 \\
& \Rightarrow \tilde{A}<\tilde{B}
\end{aligned}
$$

It is observed that the above ranking order is unaltered even by using the index of modality proposed in section 3 irrespective of decision maker.

\section{COMPARATIVE STUDY}

Example 5.1 Consider four normalized trapezoidal fuzzy numbers

$$
\begin{aligned}
& \tilde{A}_{1}=(0.1,0.2,0.3 ; 1), \tilde{A}_{2}=(0.2,0.5,0.8 ; 1), \\
& \tilde{A}_{3}=(0.3,0.4,0.9 ; 1), \tilde{A}_{4}=(0.6,0.7,0.8 ; 1)
\end{aligned}
$$

which were ranked earlier by Yager[8], Fortemps and Roubens[27], Liou and Wang[25], and Chen and Lu [33] as shown in Table I.

It can be seen from Table I that none of the methods discriminates fuzzy numbers. Yager[8] and Fortemps and Roubens [27] methods failed to discriminate the fuzzy numbers $\tilde{A}_{2}$ and $\tilde{A}_{3}$, whereas the methods of Liou and Wang[25], and Chen and Lu [33] cannot discriminate the fuzzy numbers $\tilde{A}_{2}, \tilde{A}_{3}$ and $\tilde{A}_{1}, \tilde{A}_{4}$.

By using our method, we have

$$
\begin{aligned}
& O_{\widetilde{A}_{1}}\left(\bar{x}_{0}, \bar{y}_{0}\right)=(0.2,0.33), \\
& O_{\widetilde{A}_{2}}\left(\bar{x}_{0}, \bar{y}_{0}\right)=(0.5,0.3633), \\
& O_{\widetilde{A}_{3}}\left(\bar{x}_{0}, \bar{y}_{0}\right)=(0.4,0.35), \\
& O_{\widetilde{A}_{4}}\left(\bar{x}_{0}, \bar{y}_{0}\right)=(0.7,0.33) .
\end{aligned}
$$

Therefore,

$$
\begin{aligned}
& R\left(\tilde{A}_{1}\right)=0.3858, R\left(\tilde{A}_{2}\right)=0.6161, \\
& R\left(\tilde{A}_{3}\right)=0.5315, R\left(\tilde{A}_{4}\right)=0.7738 \\
& \Rightarrow \tilde{A}_{4}>\tilde{A}_{2}>\tilde{A}_{3}>\tilde{A}_{1}
\end{aligned}
$$

It is observed that the above ranking order is unaltered even by using the index of modality proposed in section 3 irrespective of decision maker.

Example 5.2 Let $\tilde{A}=(0.1,0.2,0.4,0.5 ; 1)$ be a normalized trapezoidal fuzzy number and $\widetilde{B}=(1,1,1,1 ; 1)$ be a crisp number.

Cheng [28] ranked fuzzy numbers with the distance method using the Euclidean distance between the Centroid point and original point.

whereas Chu and Tsao [34] proposed a ranking function which is the area between the centroid point and original point . Their centroid formulae are given by

$$
\left(\bar{x}_{0}, \bar{y}_{0}\right)
$$




$$
\begin{aligned}
& =\left(\frac{w\left(d^{2}-2 c^{2}+2 b^{2}-a^{2}+d c-a b\right)+3\left(c^{2}-b^{2}\right)}{3 w(d-c+b-a)+6(c-b)},\right. \\
& \left.\frac{w}{3}\left(1+\frac{(b+c)-(a+d)(1-w)}{(b+c-a-d)+2(a+d) w}\right)\right) \\
& \left(\bar{x}_{0}, \bar{y}_{0}\right)= \\
& \left(\frac{w\left(d^{2}-2 c^{2}+2 b^{2}-a^{2}+d c-a b\right)+3\left(c^{2}-b^{2}\right)}{3 w(d-c+b-a)+6(c-b)},\right. \\
& \left.\frac{w}{3}\left(1+\frac{(b+c)}{a+b+c+d}\right)\right)
\end{aligned}
$$

Example 5.3 Let $\tilde{A}=(3,5,7 ; 1), \tilde{B}=\left(4,5, \frac{51}{8} ; 1\right)$ be two normalized triangular fuzzy numbers.

$$
\begin{aligned}
& \text { Then } O_{\widetilde{A}}\left(\bar{x}_{0}, \bar{y}_{0}\right)=(5,1.6666) \text { and } \\
& O_{\widetilde{B}}\left(\bar{x}_{0}, \bar{y}_{0}\right)=(5,0.7916)
\end{aligned}
$$

Therefore,

$$
R(\tilde{A})=5.2683 \text { and } R(\tilde{B})=5.0622 \Rightarrow \tilde{A}>\tilde{B}
$$

It is observed that the above ranking order is unaltered even by using the index of modality proposed in section 3 irrespective of decision maker.

Example 5.4 Let $\tilde{A}=(0.1,0.3,0.5 ; 1)$ be a normalized triangular fuzzy number and $\widetilde{B}=(1,1,1,1 ; 1)$ be a crisp number.

Cheng [28] ranked fuzzy numbers with the distance method using the Euclidean distance between the Centroid point and original point. Whereas Chu and Tsao [34] proposed a ranking function which is the area between the centroid point and original point . Their centroid formulae are given by

$$
\begin{aligned}
& \left(\bar{x}_{0}, \bar{y}_{0}\right)= \\
& \left(\frac{w\left(d^{2}-2 c^{2}+2 b^{2}-a^{2}+d c-a b\right)+3\left(c^{2}-b^{2}\right)}{3 w(d-c+b-a)+6(c-b)},\right. \\
& \left.\frac{w}{3}\left(1+\frac{(b+c)-(a+d)(1-w)}{(b+c-a-d)+2(a+d) w}\right)\right)
\end{aligned}
$$

Both these centroid formulae cannot rank crisp numbers which are a special case of fuzzy numbers as it can be seen from the above formulae that the denominator in the first coordinate of their centroid formulae is zero, and hence centroid of crisp numbers are undefined for their formulae. By using our method, we have $O_{\tilde{A}}\left(\bar{x}_{0}, \bar{y}_{0}\right)=(0.3,0.44)$ and $O_{\tilde{B}}\left(\bar{x}_{0}, \bar{y}_{0}\right)=(1,0.3333)$.

Therefore,

$$
R(\tilde{A})=0.5325 \text { and } R(\tilde{B})=1.0440 \Rightarrow \tilde{A}<\tilde{B}
$$

It is observed that the above ranking order is unaltered even by using the index of modality proposed in section 3 irrespective of the decision maker.

$$
\begin{aligned}
& \left(\bar{x}_{0}, \bar{y}_{0}\right)= \\
& \left(\frac{w\left(d^{2}-2 c^{2}+2 b^{2}-a^{2}+d c-a b\right)+3\left(c^{2}-b^{2}\right)}{3 w(d-c+b-a)+6(c-b)},\right. \\
& \left.\frac{w}{3}\left(1+\frac{(b+c)}{a+b+c+d}\right)\right)
\end{aligned}
$$

Both these centroid formulae cannot rank crisp numbers which are a special case of fuzzy numbers as it can be seen from the above formulae that the denominator in the first coordinate of their centroid formulae is zero, and hence centroid of crisp numbers are undefined for their formulae. By using our method, we have

$$
\begin{aligned}
& O_{\widetilde{A}}\left(\bar{x}_{0}, \bar{y}_{0}\right)=(0.3,0.3466) \text { and } \\
& O_{\widetilde{B}}\left(\bar{x}_{0}, \bar{y}_{0}\right)=(1,0.3333)
\end{aligned}
$$

Therefore,

$$
R(\tilde{A})=0.4583 \text { and } R(\tilde{B})=1.0540 \Rightarrow \tilde{A}<\tilde{B}
$$

It is observed that the above ranking order is unaltered even by using the index of modality proposed in section 3 irrespective of the decision maker.

\section{APPLICATION OF THE PROPOSED FUZZY RANKING METHOD TO FUZZY RISK ANALYSIS}

In this section, the proposed fuzzy method is applied to fuzzy risk analysis problems.

Let $C_{1}, C_{2}, \ldots, C_{n}$ be $\mathrm{n}$ manufactures producing the products respectively. Suppose, the product $\mathrm{A}_{\mathrm{i}}$ produced by the manufacturer $\mathrm{C}_{\mathrm{i}}$ is composed of $\mathrm{p}$ sub-components 
$A_{i 1}, A_{i 2}, \ldots, A_{i p}, 1 \leq i \leq n$. To know the probability of failure of component $\tilde{\mathrm{R}}_{\mathrm{i}}$ of component $A_{i}$ produced by the manufacture $C_{i}$, we use the evaluating terms $\tilde{R}_{i k}$ and $\tilde{\mathrm{W}}_{\mathrm{ik}}$ respectively called the probability of failure and severity of loss of the sub-component $A_{i k}, 1 \leq k \leq p$. The structure of the fuzzy risk is shown in Fig. 2

The nine-member linguistic term set is shown in Table II is used for representing the linguistic terms and their corresponding generalized fuzzy numbers respectively. Each linguistic term shown in Table II is defined in the universe of discourse $[0,1]$.

The algorithm to deal with fuzzy risk analysis is presented as follows:

Step 1: Find the probability of failure $\tilde{R}_{i}$ of each component $\tilde{\mathrm{A}}_{\mathrm{i}}$ made by manufacturer $\tilde{C}_{i}$, where $1 \leq i \leq n$ by using the fuzzy weighted mean method and the generalized fuzzy number arithmetic operations to integrate the factors of each sub-component $\tilde{A}_{i k}$ where $1 \leq k \leq p$,i.e., $\quad \tilde{R}_{i}=\sum_{k=1}^{p}\left(\tilde{W}_{i k} \otimes \tilde{R}_{i k}\right)$

$\varnothing \sum_{k=1}^{p} \tilde{W}_{i k}$ where $\tilde{R}_{i}$ is a generalized fuzzy number given by $\tilde{R}_{i}=\left(r_{i 1}, r_{i 2}, r_{i 3}, r_{i 4} ; w_{\tilde{R}_{i}}\right)$.

Step 2: Find the orthocenter of centroids and ranking indices $R\left(\widetilde{R}_{1}\right), R\left(\widetilde{R}_{2}\right), R\left(\widetilde{R}_{3}\right), \ldots, R\left(\widetilde{R}_{n}\right) \quad$ of each fuzzy number $\tilde{R}_{1}, \tilde{R}_{2}, \ldots, \tilde{R}_{n}$ by using $O_{\tilde{A}}\left(\bar{x}_{0,} \bar{y}_{0}\right)=\left(\begin{array}{l}\frac{1}{2}(c+b), \\ \frac{(3 c-2 a-b)(c-3 b+2 d)+2 w^{2}}{6 w}\end{array}\right)$ and $R(\tilde{A})=\sqrt{\bar{x}_{0}^{2}+\bar{y}_{0}^{2}}$.

The larger the value of $R\left(\tilde{R}_{i}\right)$, the higher is the probability of failure of component $A_{i}$ made by the manufacturer $C_{i}$.

Step 3: In case when the ranking indices $R\left(\widetilde{R}_{1}\right), R\left(\widetilde{R}_{2}\right), R\left(\widetilde{R}_{3}\right), \ldots, R\left(\tilde{R}_{n}\right)$ of the fuzzy numbers $\widetilde{R}_{1}, \widetilde{R}_{2}, \ldots, \widetilde{R}_{n}$ are equal, then use the cases (i) to (iii) in section 3 to find the ranking index of the fuzzy numbers which involves the decision maker's optimistic attitude along with the importance of central value and spreads. The larger the value of $R\left(\widetilde{R}_{i}\right)$, the higher is the probability of failure of component $A_{i}$, made by the manufacturer $C_{i}$.

To illustrate the fuzzy risk analysis of the proposed method, let $C_{1}, \mathrm{C}_{2}$ and $C_{3}$ be three manufacturers producing the products $A_{1}, A_{2}$ and $A_{3}$ respectively. Suppose, the product $\mathrm{A}_{\mathrm{i}}$ produced by the manufacturer $C_{i}$ is composed of three sub-components $A_{i 1}, A_{i 2}$ and $A_{i 3}, 1 \leq i \leq 3$. To know the probability of failure $\tilde{R}_{i}$ of the component $A_{i}$ produced by the manufacturer $C_{i}$. We use the evaluating terms $\tilde{R}_{i k}$ and $\tilde{W}_{i k}$ respectively, called the probability of failure and severity of loss of the subcomponent $A_{i k}, 1 \leq k \leq 3$. The nine-member linguistic term set shown in Table II is used to represent the linguistic terms and their corresponding generalized fuzzy numbers, respectively. The linguistic values of the evaluating terms $\tilde{R}_{i k}$ and $\tilde{W}_{i k}$ of the sub-components $A_{i k}$ made by the manufacturer $C_{i}$ are shown in Table III [10] .In the last column of Table III, $W_{\widetilde{R}_{i k}}$ denotes the degree of confidence of the decision maker's opinion with respect to the sub-component $A_{i k}$ made by the manufacturer $C_{i}$, where $1 \leq i \leq 3$ and $1 \leq k \leq 3$.

For the above problem, the fuzzy risk analysis is evaluated by using the proposed method as: Step1: Combine the evaluating items $\tilde{R}_{i k}$ and $\tilde{W}_{i k}$ of sub-components $A_{i k}$ of each component $A_{i}$, made by the manufacturer $C_{i}$ by using the fuzzy arithmetic operations, where $1 \leq k \leq p$ and $1 \leq i \leq n$, are given by $\widetilde{R}_{1}=(0.1659,0.2803,0.74631,1.1545 ; 0.7) ;$ $\widetilde{R}_{2}=(0.3221,0.4949,1.13921,1.6373 ; 0.85) ;$ $\widetilde{R}_{3}=(0.3659,0.51341,1.1189,1.5984 ; 0.8)$.

Step2: Using

$$
\begin{aligned}
& O_{\tilde{A}}\left(\bar{x}_{0,} \bar{y}_{0}\right)=\left(\begin{array}{l}
\frac{1}{2}(c+b), \\
\frac{(3 c-2 a-b)(c-3 b+2 d)+2 w^{2}}{6 w}
\end{array}\right) \\
& R(\tilde{A})=\sqrt{\bar{x}_{0}^{2}+\bar{y}_{0}^{2}}
\end{aligned}
$$

the orthocenter of centroids of each fuzzy number. 
$\widetilde{R}_{1}, \widetilde{R}_{2}$ and $\widetilde{R}_{3}$ and their respective ranking indexes are calculated, shown as follows:

$O_{\widetilde{R}_{1}}\left(\tilde{x}_{0}, \tilde{y}_{0}\right)=(0.5133,1.0910) ; R\left(\widetilde{R}_{1}\right)=1.2057$, $O_{\widetilde{R}_{2}}\left(\tilde{x}_{0}, \tilde{y}_{0}\right)=(0.8170,1.5919) ; R\left(\tilde{R}_{2}\right)=1.7893$, $O_{\widetilde{R}_{3}}\left(\tilde{x}_{0}, \tilde{y}_{0}\right)=(0.8161,1.4875) ; R\left(\widetilde{R}_{3}\right)=1.6966$, and the ranking order of the fuzzy numbers $\tilde{\mathrm{R}}_{1}, \tilde{\mathrm{R}}_{2}$ and $\tilde{\mathrm{R}}_{3}$ is $\tilde{R}_{2}>\widetilde{R}_{3}>\tilde{R}_{1}$. That is, the ranking order of the risk of the manufacturer $\mathrm{C}_{1}, \mathrm{C}_{2}$ and $C_{3}$ is $C_{2}>C_{3}>C_{1}$. That is, the component $A_{2}$ made by the Manufacturer $C_{2}$ has the highest probability of failure.

\section{CONCLUSIONS}

Many approaches in ranking fuzzy numbers fail to clearly discriminate the fuzzy numbers. Therefore, in this paper a ranking method based on orthocenter of centroids is proposed and a new method to deal with fuzzy risk analysis is presented which is based on ranking fuzzy numbers. First, a new method to rank fuzzy numbers is proposed. The proposed fuzzy ranking method overcomes some of the drawbacks of the existing methods as they failed to discriminate fuzzy numbers and could not rank crisp numbers which are a special case of fuzzy numbers. Another important feature of the proposed fuzzy ranking method is that, it takes into consideration the decision maker optimistic attitude as well as the index of modality which tells the importance of central value and spreads while ranking fuzzy numbers. We also applied the proposed fuzzy ranking method to deal with fuzzy risk analysis, where the evaluating values are represented by fuzzy numbers. The proposed method is useful method to deal with fuzzy risk analysis as it considers the decision maker's view in both the stages.

Table I. Comparison of various ranking methods

\begin{tabular}{|c|c|c|c|c|c|c|}
\hline Fuzzy ranking & & $\tilde{A}_{1}$ & $\tilde{A}_{2}$ & $\tilde{A}_{3}$ & $\tilde{A}_{4}$ & Ranking order \\
\hline Yager [8] & & 0.20 & 0.50 & 0.50 & 0.70 & $\tilde{A}_{4}>\tilde{A}_{2} \approx \tilde{A}_{3}>\tilde{A}_{1}$ \\
\hline Fortemps\&Roubens[27] & & 0.20 & 0.50 & 0.50 & 0.70 & $\tilde{A}_{4}>\tilde{A}_{2} \approx \tilde{A}_{3}>\tilde{A}_{1}$ \\
\hline \multirow{3}{*}{ Liou \& Wang[25] } & $\alpha=1$ & 0.25 & 0.65 & 0.65 & 0.75 & $\tilde{A}_{4}>\tilde{A}_{2} \approx \tilde{A}_{3}>\tilde{A}_{1}$ \\
\hline & $\alpha=0.5$ & 0.20 & 0.50 & 0.50 & 0.70 & $\tilde{A}_{4}>\tilde{A}_{2} \approx \tilde{A}_{3}>\tilde{A}_{1}$ \\
\hline & $\alpha=0$ & 0.15 & 0.35 & 0.35 & 0.65 & $\tilde{A}_{4}>\tilde{A}_{2} \approx \tilde{A}_{3}>\tilde{A}_{1}$ \\
\hline \multirow{3}{*}{ Chen [33] } & $\beta=1$ & -0.20 & 0.00 & 0.00 & -0.20 & $\tilde{A}_{2} \approx \tilde{A}_{3}>\tilde{A}_{1} \approx \tilde{A}_{4}$ \\
\hline & $\beta=0.5$ & -0.20 & 0.00 & 0.00 & -0.20 & $\tilde{A}_{2} \approx \tilde{A}_{3}>\tilde{A}_{1} \approx \tilde{A}_{4}$ \\
\hline & $\beta=0$ & -0.20 & 0.00 & 0.00 & -0.20 & $\tilde{A}_{2} \approx \tilde{A}_{3}>\tilde{A}_{1} \approx \tilde{A}_{4}$ \\
\hline Proposed method & & 0.39 & 0.62 & 0.53 & 0.77 & $\tilde{A}_{4}>\tilde{A}_{2}>\tilde{A}_{3}>\tilde{A}_{1}$ \\
\hline
\end{tabular}




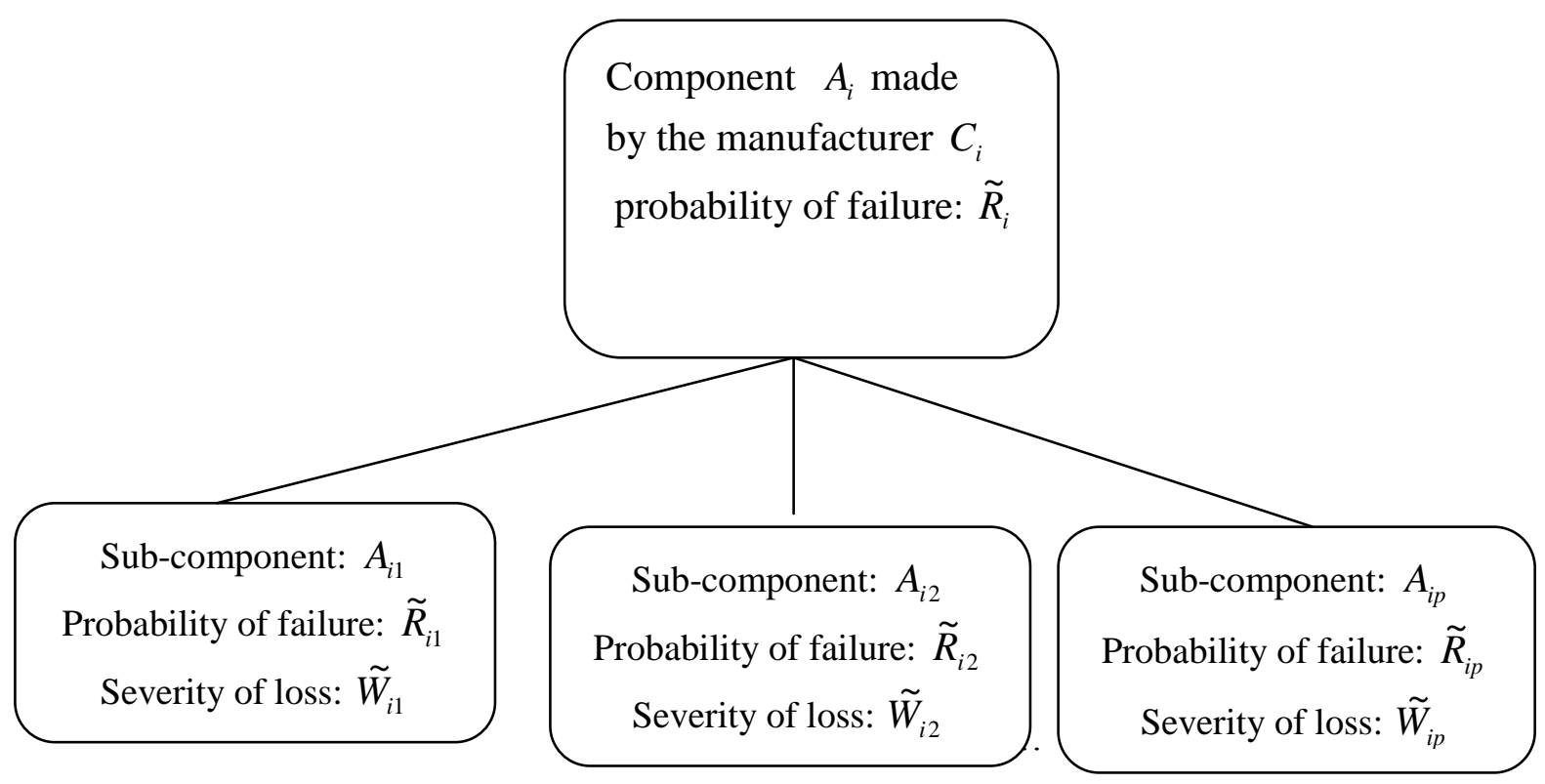

Figure2: The structure of fuzzy risk analysis

Table II: Linguistic terms and their corresponding generalized fuzzy numbers

\begin{tabular}{|c|c|}
\hline Linguistic terms & Generalized fuzzy numbers \\
\hline Absolutely-low & $(0.0,0.0,0.0,0.0 ; 1.0)$ \\
\hline Very-low & $(0.0,0.0,0.02,0.07 ; 1.0)$ \\
\hline Low & $(0.04,0.10,0.18,0.23 ; 1.0)$ \\
\hline Fairly-low & $(0.17,0.22,0.36,0.42 ; 1.0)$ \\
\hline Medium & $(0.32,0.41,0.58,0.65 ; 1.0)$ \\
\hline Fairly-high & $(0.58,0.63,0.80,0.86 ; 1.0)$ \\
\hline High & $(0.72,0.78,0.92,0.97 ; 1.0)$ \\
\hline Very-high & $(0.93,0.98,1.0,1.0 ; 1.0)$ \\
\hline Absolutely-high & $(1.0,1.0,1.0,1.0 ; 1.0)$ \\
\hline
\end{tabular}

Table III : Linguistic values of the evaluating items of the sub-components made by the manufacturer

\begin{tabular}{|c|l|l|l|}
\hline Manufacturer & Subcomponents & $\begin{array}{l}\text { Linguistic values of } \\
\text { the severity of loss }\end{array}$ & \multicolumn{1}{|c|}{$\begin{array}{c}\text { Linguistic values of the } \\
\text { probability of failure }\end{array}$} \\
\hline \multirow{3}{*}{$C_{1}$} & $A_{11}$ & $\tilde{W}_{11}=$ low & $\tilde{R}_{11}$ fairly-low $\left(\tilde{W}_{\widetilde{R}_{11}}=0.9\right)$ \\
\cline { 2 - 4 } & $A_{12}$ & $\tilde{W}_{12}=$ fairly-high & $\tilde{R}_{12}=$ medium $\left(\tilde{W}_{\tilde{R}_{12}}=0.7\right)$ \\
\cline { 2 - 4 } & $A_{13}$ & $\tilde{W}_{13}$ =very-low & $\tilde{R}_{13}=$ fairly-high $\left(\tilde{W}_{\tilde{R}_{13}}=0.8\right)$ \\
\hline \multirow{3}{*}{$C_{2}$} & $A_{21}$ & $\tilde{W}_{21}=$ low & $\tilde{R}_{21}=$ very-high $\left(\tilde{W}_{\tilde{R}_{21}}=0.85\right)$ \\
\cline { 2 - 4 } & $A_{22}$ & $\tilde{W}_{22}=$ fairly-high & $\tilde{R}_{22}=$ fairly-high $\left(\tilde{W}_{\tilde{R}_{22}}=0.9\right)$ \\
\hline
\end{tabular}




\begin{tabular}{|l|l|l|l|}
\hline & $A_{23}$ & $\tilde{W}_{23}$ =very-low & $\tilde{R}_{23}=$ medium $\left(\tilde{W}_{\widetilde{R}_{23}}=0.9\right)$ \\
\hline \multirow{3}{*}{$C_{3}$} & $A_{31}$ & $\tilde{W}_{31}=$ low & $\tilde{R}_{31}=$ fairly-low $\left(\tilde{W}_{\tilde{R}_{31}}=0.95\right)$ \\
\cline { 2 - 4 } & $A_{32}$ & $\tilde{W}_{32}=$ fairly-high & $\tilde{R}_{32}=\operatorname{high}\left(\tilde{\mathrm{W}}_{\tilde{\mathrm{R}}_{32}}=0.8\right)$ \\
\cline { 2 - 4 } & $A_{33}$ & $\tilde{W}_{33}=$ very-low & $\tilde{R}_{33}=$ fairly-high $\left(\tilde{\mathrm{W}}_{\tilde{\mathrm{R}}_{33}}=1.0\right)$ \\
\hline
\end{tabular}

\section{REFERENCES}

[1] L.A. Zadeh, Fuzzy sets, Information and control 8 (1965) 338-353.

[2] R. Jain, Decision making in the presence of fuzzy variables, IEEE Trans. on Sys., Man and Cybernetics 6 (1976) 698-703.

[3] S.M. Bass, H. Kwakernaak, Rating and ranking of multiple-aspect alternatives using Fuzzy sets, Automatica 13 (1977) 47-58.

[4] R. Jain, A procedure for multi aspect decision making using fuzzy sets. Int. J. Syst. Sci. 8 (1978) $1-7$.

[5] J. F. Baldwin, N.C.F Guild, Comparison of fuzzy sets on the same decision space,Fuzzy Sets and Systems 2 (1979) 213-233.

[6] R.R. Yager, On choosing between fuzzy subsets, Kybernetes 9 (1980) 151-154.

[7] J.M. Adamo, Fuzzy decision trees, Fuzzy Sets and Systems 4 (1980) 207-219

[8] R. R. Yager, A procedure for ordering fuzzy subsets of the unit interval, Information Sciences 24 (1981) 143-161.

[9] W. Chang, Ranking of fuzzy utilities with triangular membership functions, Proceedings of International Conference on Policy Analysis and Systems, 1981, pp. 263-272.

[10] E. Kerre, The use of fuzzy set theory in electrocardiological diagnostics, in: M.M. Gupta, E. Sanchez (Eds.), Approximate Reasoning in Decision-Analysis, North- Holland, Amsterdam, 1982, pp. 277-282.

[11] D. Dubois and H. Prade, Ranking fuzzy numbers in the setting of possibility theory, Information Sciences 30 (1983) 183-224.

[12] G. Bortolan, R. Degani, A review of some methods for ranking fuzzy subsets, Fuzzy Sets and Systems 15 (1985) 1-19.

[13] S.-H. Chen, Ranking fuzzy numbers with maximizing set and minimizing set, Fuzzy Sets and Systems 17 (1985) 113-129.

[14] W. Kolodziejczyk, Orlovsky, Concept of decisionmaking with fuzzy preference relation - Further results, Fuzzy Sets and Systems 19 (1986) 11-20.

[15] K. Nakamura, Preference relations on a set of fuzzy utilities as a basis for decision making. Fuzzy Sets and Systems 20 (1986) 147-162.

[16] D. Dubois, H. Prade, The mean value of a fuzzy number, Fuzzy Sets and Systems 2 (1987) 279-300.
[17] X. Wang, A class of approaches to ordering alternatives, MSc Thesis, Taiyuan University Technology, 1987 (in Chinese).

[18] E. S. Lee, R.-J. Li, Comparison of fuzzy numbers based on the probability measure of fuzzy events, Comput. Math. Applic. 15(10) (1988) 887-896.

[19] M. Delgado, J.L. Verdegay, M.A. Vila, A procedure for ranking fuzzy numbers, Fuzzy Sets and Systems 26 (1988) 49-62.

[20] L. Campos, A. Munoz, A subjective approach for ranking fuzzy numbers, Fuzzy Sets and Systems 29 (1989) 145-153.

[21] K. Kim, K. S. Park, Ranking fuzzy numbers with index of optimism, Fuzzy Sets and Systems 35 (1990) 143-150.

[22] Y. Yuan, Criteria for evaluating fuzzy ranking methods, Fuzzy Sets and Systems 44 (1991) 139157.

[23] 23. S. Heilpern, The expected value of a fuzzy number, Fuzzy Sets and Systems 47 (1992) 8186.

[24] J. J. Saade, H. Schwarzlander, Ordering fuzzy sets over the real line: An approach based on decision making under uncertainty, Fuzzy Sets and Systems 50 (1992) 237-246.

[25] T. S. Liou, M. - J. Wang, Ranking fuzzy numbers with integral value, Fuzzy Sets and Systems 50 (1992) 247-255.

[26] F. Choobineh H. Li, An index for ordering fuzzy numbers, Fuzzy Sets and Systems 54 (1993) 287294.

[27] P.Fortemps, M.Roubens, Ranking and defuzzification methods based on area compensation, Fuzzy Sets and Systems 82 (1996) 319-330

[28] C. H. Cheng, A new approach for ranking fuzzy numbers by distance method, Fuzzy Sets and Systems 95 (1998) 307-317.

[29] J.-S. Yao. K. Wu, Ranking fuzzy numbers based on decomposition principle and signed distance, Fuzzy Sets and Systems 116 (2000) 275-288.

[30] X. Wang, E. E. Kerre, Reasonable properties for the ordering of fuzzy quantities (I), Fuzzy Sets and Systems 118 (2001) 375-385.

[31] X. Wang, E. E. Kerre, Reasonable properties for the ordering of fuzzy quantities (II), Fuzzy Sets and Systems 118 (2001) 387-405. 
[32] M. Modarres, S. S.-Nezhad, Ranking fuzzy numbers by preference ratio, Fuzzy Sets and Systems 118 (2001) 429-436.

[33] L.-H. Chen, H.-W. Lu, An approximate approach for ranking fuzzy numbers based on left and right dominance, Computers and Mathematics with Applications

(2001) 1589-1602.

[34] T.-C. Chu, C. - T. Tsao, Ranking fuzzy numbers with an area between the Centroid point and original point, Computers and Mathematics with Applications 43 (2002) 111- 117.

[35] Y.- M. Wang, J.- B. Yang, D.- L. Xu, K.- S. Chin, On the centroids of fuzzy numbers, Fuzzy Sets and Systems 157 (2006) 919-926.

[36] S. Abbasbandy, B. Asady, Ranking of fuzzy numbers by sign distance, Information Sciences 176 (2006) 2405-2416.

[37] B. Asady, A. Zendehnam, Ranking fuzzy numbers by distance minimization, Applied Mathematical Modelling 31 (2007) 2589-2598.

[38] M. S. Garcia, M. T. Lamata, A modification of the index of liou and wang for ranking fuzzy number, International Journal of Uncertainty, Fuzziness and Knowledge-Based Systems 15 (4) (2007) 411-424.

[39] Y.-J. Wang, H.-S. Lee, The revised method of ranking fuzzy numbers with an area between the centroid and original points, Computers and Mathematics with Applications 55 (2008) 20332042.

[40] C.-C. Chen, H.-C. Tang, Ranking nonnormal pnorm trapezoidal fuzzy numbers with integral value, Computers and Mathematics with Applications 56 (2008) 2340-2346.

[41] S.H. Chen, Operations on fuzzy numbers with function principal, Tamkang J. Manag. Sci., Vol.6, no.1, pp.13-25, 1985.

[42] Kaufmann, A. and Gupta, M.M. (1985), Introduction to Fuzzy arithmetics : Theory

[43] and applications, Van Nostrand Reinhold, New York.

[44] C-H. Hsieh, S-H Chen, A model and algorithm of fuzzy product positioning; Information Sciences , vol.121, no. 12, pp.61-82,1999. 